\title{
Optimization of breath-holding time for intrahepatic fat quantification using proton-magnetic resonance spectroscopy at 1.5-Tesla magnetic resonance imaging
}

\section{Yuhei Otsuka ( $\nabla$ hey.u3289@gmail.com )}

Tokai University Oiso Hospital: Tokai Daigaku Igakubu Fuzoku Oiso Byoin https://orcid.org/00000003-3220-3702

\section{Shiho Sakamura}

Tokai University Oiso Hospital: Tokai Daigaku Igakubu Fuzoku Oiso Byoin Kazuhiro Watanabe

Tokai University Oiso Hospital: Tokai Daigaku Igakubu Fuzoku Oiso Byoin

\section{Research Article}

Keywords: Magnetic Resonance Imaging, Proton Magnetic Resonance Spectroscopy, intrahepatic fat quantification, point resolved spectroscopy, Breath Holding, Delivery of Health Care

Posted Date: July 22nd, 2021

DOl: https://doi.org/10.21203/rs.3.rs-692861/v1

License: (c) (i) This work is licensed under a Creative Commons Attribution 4.0 International License. Read Full License 


\section{Abstract}

Purpose We determined the minimum required breath-holding time (Modified ${ }^{1} \mathrm{H}-\mathrm{MRS}$ ) and conducted a backward study on patients who underwent intrahepatic fat quantification using the point resolved spectroscopy method. We investigated the correlation between dual echo-MRI (DE-MRI) and liver attenuation index ( $\mathrm{LAl}$ ) measurements and evaluated the validity of the analysis results to determine whether breath-holding time (BHT) could be reduced.

Methods In the MRS-fat fraction (MRS-FF) analysis, using only the reference datasets, the minimum required breath-holding time was set to $14 \mathrm{~s}$. To evaluate the validity of the obtained MRS-FF, we compared the correlation using the dual echo-fat fraction (DE-FF) and LAl as a reference values, the correlation coefficient was investigated by obtaining the Spearman's rank correlation coefficient. Next, we compared the correlation in two subgroups of the abovementioned case groups: (1) those with a BHT of $23 \mathrm{~s}$ and (2) those with a BHT between 14 to $22 \mathrm{~s}$.

Results The correlation coefficients between MRS-FF and DE-FF and MRS-FF and LAI were 0.841 ( $p=$ $0.00)$ and $-0.726(p<0.01)$, respectively. Next, in the subgroup analysis, the correlation coefficients of MRS-FF and DE-FF and MRS-FF and LAI were $0.849(p=0.00)$ and $-0.733(p=0.00)$, respectively, in group (1) and $0.886(p=0.00)$ and $-0.761(p=0.00)$, respectively, in group (2).

Conclusion In the imaging using Modified ${ }^{1} \mathrm{H}-\mathrm{MRS}$, the present method suggested that the reduction in breath-holding time from 14 to $22 \mathrm{~s}$ did not affect the MRS-FF analysis results.

\section{Introduction}

Recent studies have shown that the incidence of non-virologic $B$ and $C$ liver cancers is increasing. In previous studies, the incidence of non-B and non-C hepatocellular carcinoma in Japan from 1991 to 2015 has shown an increasing trend, from $10 \%$ in 1991 to $24.1 \%$ in 2010 and $32.5 \%$ in 2015 [1,2]. Moreover, previous studies reported that there is a higher incidence of obesity and lifestyle diseases compared to viral hepatitis-associated hepatocellular carcinoma, but more than half of the patients are diagnosed in an advanced state [3]. Among them, there is an increased incidence of non- $B$ and non-C hepatocellular carcinoma caused by non-alcoholic fatty liver disease (NAFLD) and non-alcoholic steatohepatitis. Unlike virus-related hepatocellular carcinomas, for which the high-risk population and surveillance programs are well established, there are limited studies on the characteristics of these non-B- and non-C-type hepatocellular carcinomas. In the pathogenesis and progression of NAFLD, fat accumulation in the liver is the basis of pathogenesis, from which inflammation, fibrosis, and carcinogenesis progress $[4,5]$. Therefore, it is essential to detect the liver lipid deposition accurately and early by clinical examination. In Japan, the number of subjects with metabolic syndrome has been increasing, and data from medical and physical examinations have shown that $30 \%$ of the Japanese population have complications of fatty liver $[6,7,8]$. For the determination of intrahepatic fat, various imaging modalities, such as ultrasound and computed tomography (CT), have been developed, and magnetic resonance imaging (MRI) such as 3D 
T1WI dual echo (DE) has the highest sensitivity and specificity. Particularly, MRS fat fraction (MRS-FF) measured by proton-magnetic resonance spectroscopy ( ${ }^{1} \mathrm{H}-\mathrm{MRS}$ ) correlates well with histopathological evaluation and can quantify the liver fat content, which is referred to as the "gold standard" alternative to histopathological evaluation $[9,10,11]$. A previous study by Rastogi et al. revealed the correlation coefficients between ${ }^{1} \mathrm{H}$-MRS and CT (liver attenuation index [LAI]) and DE-MRI besides the correlation between ${ }^{1} \mathrm{H}$-MRS and histopathological evaluation [12]. Recently, proton density fat fraction using the multipoint-Dixon method has been released commercially, but it can only be used with newer devices and is insufficiently versatile $[13,14]$.

Conversely, ${ }^{1} \mathrm{H}-\mathrm{MRS}$ using the single-shot-point resolved spectroscopy (PRESS) method is commonly used in a general clinical application. In ${ }^{1} \mathrm{H}$-MRS for head tumors, water suppression is used because the water signal in the voxel disturbs specter analysis, but for liver fat quantification, the analysis of the specter peaks of water and lipid is necessary. However, because of the long scanning time, it is difficult to apply to patients who have difficulty in breath holding for a long time. Previous studies have not found the minimum required breath-holding time for the purpose of expanding the use of ${ }^{1} \mathrm{H}-\mathrm{MRS}$ imaging to more patients. Therefore, we defined a minimum breath-holding time condition (Modified ${ }^{1} \mathrm{H}-\mathrm{MRS}$ ) and examined the correlation with DE-MRI and CT (LAI) results in patients who underwent intrahepatic fat determination using the PRESS method. The results of the analysis were validated to determine whether it is possible to reduce the breath-holding time.

\section{Materials And Methods}

\section{Selection of Subjects}

This retrospective study was approved by the Institutional Review Board No. 20R-399 of Tokai University Oiso Hospital. The study period was from August 1, 2015 to May 31, 2020. In this study, we used 3D T1WI DE-MRI and ${ }^{1} \mathrm{H}$-MRS specter data of patients who underwent non-contrast liver MRI for intrahepatic fat quantification, and CT images of patients who underwent non-contrast CT for visceral fat quantification. Data were extracted from 58 patients who underwent ${ }^{1} \mathrm{H}$-MRS scanning and CT scan on the same day. 3D T1WI DE-MRI and non-contrast CT provided axial images, and ${ }^{1} \mathrm{H}-\mathrm{MRS}$ was performed with single-voxel acquisition. However, two patients with iron deposition in the liver and two aged $<18$ years were excluded from the extracted data. Therefore, the final number of patients was 54 . The included patients did not receive any interventions before or after this study. The subjects in this dataset required liver MRI, ${ }^{1} \mathrm{H}-\mathrm{MRS}$, and abdominal $\mathrm{CT}$ at the discretion of their physicians, and the scans were performed by radiologists under optimal imaging conditions. When extracting patient data, we informed patients on the bulletin board and website that anonymized MRI, ${ }^{1} \mathrm{H}-\mathrm{MRS}$ specter data, and CT images may be used in this study. Simultaneously, they were informed that they could opt out of this study. As a result, zero subjects disagreed to join the study.

\section{Analysis Procedure}


For DE-MRI and CT (LAI), region of interest (ROI) settings and value measurements were obtained using PACS viewer: ShadeQuest/ViewR version 1.22.85 (Yokogawa Medical Solutions Corporation, Tokyo, Japan).

The MRS-FF calculated from the MRS analysis was calculated using Eq. (1) using the ratio of area under the peak (AUP) at the specter peak of water to AUP at the specter peak of intrahepatic lipid [9-12].

MRS-FF $[\%]=\left(\mathrm{AUP}_{\text {Lipid }} /\left(\mathrm{AUP}_{\text {Water }}+\mathrm{AUP}_{\text {Lipid }}\right)\right) \times 100$

In the comparison of MRI examinations, we used in-phase (IN) and opposed-phase (OP) images acquired by 3D T1WI DE-MRI, which had been scanned at the time of ${ }^{1} \mathrm{H}-\mathrm{MRS}$ examination. In both images, ROIs were set at the same location as the voxel position set in the right lobe of the liver by MRS. The ROI was set at a location avoiding blood vessels and other structures with a size of $250-350 \mathrm{~mm}^{2}$, and the dual echo-fat fraction (DE-FF) was calculated using Eq. 2 using the average signal intensity (SI) obtained from each ROI [12]:

$\mathrm{DE}-\mathrm{FF}[\%]=\left(\left(S \mathrm{I}_{\mathbb{N}}-S \mathrm{I}_{\mathrm{OP}}\right) / 2 S \mathrm{I}_{\mathbb{N}}\right) \times 100 \quad$ Eq. (2)

3D T1WI DE-MRI is the protocol that we always use for quantitative MRI of intrahepatic fat using ${ }^{1} \mathrm{H}-\mathrm{MRS}$ in our hospital. Some previous studies, other than 3D T1WI DE-MRI as the MRI protocol to be compared with ${ }^{1} \mathrm{H}$-MRS, evaluated fat deposition by the SI difference between fat saturation (FS) and non-FS images $[15,16]$. However, we did not scan fat-suppressed images in our routine protocol. Moreover, for intrahepatic fat quantification by 3D T1WI DE-MRI, d'Assignies et al. reported a correction for the T2* effect to exclude the effect of T2* shortening due to iron deposition in the liver. Besides, a study by Janakan Satkunasingham et al. validated the detection of hepatic fat in a clinical setting using the 3PD chemical shift sequence even in the presence of T2* shortening [17]. Nevertheless, 3D T1WI DE-MRI was used for comparison because the MRI system at our hospital does not allow correction for the T2* effect and use of 3PD chemical shift sequence.

As a comparison to intrahepatic fat quantification by MRI and MRS, we used slice that included the liver and spleen of non-contrast CT images obtained for visceral fat quantification to calculate the LAl, which is the difference between the average Hounsfield units $(\mathrm{HU})$ in the ROI placed in the liver and average HU in the ROI located in the spleen, using Eq. (3) [12]. ROls for the liver and spleen were set in the same locations as in DE-MRI.

$\mathrm{LAl}[\mathrm{HU}]=\mathrm{HU}_{\mathrm{ROI} \text { of liver }}-\mathrm{HU}_{\mathrm{ROI}}$ of spleen $\quad$ Eq. (3)

According to a meta-analysis reported by Bohte et al., the mean $\mathrm{HU}$ of the spleen was used as an internal reference in all studies comparing ${ }^{1} \mathrm{H}-\mathrm{MRS}$ and $\mathrm{CT}$ images for intrahepatic fat quantification.

In these study, intrahepatic fat quantification by CT images was measured using the attenuation value of the mean $\mathrm{HU}$ of the liver minus the mean $\mathrm{HU}$ of the spleen ( $\mathrm{LAl}$ ) or ratio of the mean $\mathrm{HU}$ of the liver to the 
mean HU of the spleen (L/S) [18].

\section{Subgroup Analysis}

Next, as a subgroup analysis in the case groups of 2.2, we compared the correlation between MRS-FF and DE-FF and MRS-FF and LAl in two groups: group (1) with a breath-hold time (BHT) during imaging of $23 \mathrm{~s}$ and group (2) with a BHT between 14 and $22 \mathrm{~s}$.

\section{MRI Scanner and Parameters}

The MRI system was a 1.5-Tesla MRI Signa HDxt software version.16 (GE Healthcare, Milwaukee, WI, USA), and an eight-channel body array coil (8 ch upper) was used for scanning. All ${ }^{1} \mathrm{H}-\mathrm{MRS}$ analyses were performed using Sage software version 7.1 (GE Healthcare) as the MRS analysis software. For MRS analysis, two ${ }^{1} \mathrm{H}$-MRS specter data obtained by reference datasets, 2 TR were averaged and zerodimensional correction and baseline correction, were applied to obtain the area under the specter peak of water and lipid. The scanning parameters for ${ }^{1} \mathrm{H}-\mathrm{MRS}$ were as follows: pulse sequence, Probe-P; repetition time (TR), $1149.0 \mathrm{~ms}$; echo time (TE), $27.0 \mathrm{~ms}$; number of phase-cycles (NEX), 2.00; acquisition matrix, 1 $\times 1$; average scans, 4.00 ; setting of voxels used for analysis, 1 voxel of $30 \mathrm{~mm} \times 30 \mathrm{~mm} \times 30 \mathrm{~mm}$ was set in the right lobe of the liver, and scanning was performed with breath hold (by expiratory stop). In MRS imaging, proper acquisition techniques are required to collect spectra for fat fraction estimation. Besides the PRESS method used in this study, there were other methods using the stimulated echo acquisition mode (STEAM) method. Although this STEAM method could set a shorter TE than the PRESS method, it was known that the signal-to-noise ratio (SNR) of the STEAM method was about 1/2 lower than that of the PRESS method because of the signal acquisition by stimulated echo in the STEAM method [16]. Moreover, T2 bias should be considered when using the PRESS method for intrahepatic fat quantification. T2 bias was a common confounding factor for fat quantification methods, such as the PRESS method, which employs a refocusing pulse to generate echoes. Because the minimum echo time achievable with clinically available ${ }^{1} \mathrm{H}$-MRS for intrahepatic fat determination was always greater than zero, some T2 relaxation occurs before echo sampling. As the T2 relaxation time of the intrahepatic water component was shorter than the $\mathrm{T} 2$ relaxation time of the intrahepatic fat component, there was relative enhancement in the signal from fat (i.e., longer T2 values) compared to the signal from water (i.e., shorter T2 values). To reduce the effect of this T2 bias, it was preferable to set the TE as short as possible. Therefore, compared with the STEAM method, which can select a shorter TE, the PRESS method underestimates the T2 value of intrahepatic fat and overestimates the fat fraction [25]. However, this study aimed to evaluate the MRS-FF using the minimum number of ${ }^{1} \mathrm{H}-\mathrm{MRS}$ specter data required. Therefore, we used the PRESS method because the breath-holding time when using the STEAM method was far beyond the range of a single breath-holding and because we were concerned that the repeatability of the MRS-FF would be reduced due to the lower SNR when using the STEAM method on a universal 1.5-Tesla MRI. 
In our ${ }^{1} \mathrm{H}$-MRS imaging using the PRESS method, a single ${ }^{1} \mathrm{H}-\mathrm{MRS}$ specter data was detected using the spin echo signal obtained by 1TR (Fig 1). The total scanning time was determined by the total number of frames of the presaturation pulse, dummy sets, reference datasets, and datasets (Fig 2). Default ${ }^{1} \mathrm{H}$-MRS imaging was performed in the following order: presaturation pulse, 10TR; dummy sets, 4TR; reference datasets, 2TR; datasets, 8TR; total scanning time (indicated value), 24TR (28 s). Conversely, the modified

${ }^{1} \mathrm{H}-\mathrm{MRS}$ used in this study was acquired in the following order: dummy sets, 0TR; presaturation pulse, 10TR; reference datasets, 2TR; datasets, 8TR; and total scanning time (displayed value), 20TR (23 s). In the MRS-FF analysis using Sage as the analysis software, the minimum required BHT was defined as 12 TR (14 s), which was the sum of the presaturation pulse, 10 TR, and reference datasets, 2 TR.

The scanning parameters for the 3D T1WI DE-MRI used in this study were as follows: pulse sequence, 3D FSPGR; repetition time (TR), $6.7 \mathrm{~ms}$; echo time (TE), $2.1 \mathrm{~ms} / 4.5 \mathrm{~ms}$; matrix, 256×160 (phase encode); slice thickness (scan), $4.0 \mathrm{~mm}(-2.0 \mathrm{~mm}$ ); slice thickness (MPR), $7.0 \mathrm{~mm}$; field of view, $360 \mathrm{~mm} \times 360 \mathrm{~mm}$; number of averages, 1.00; received bandwidth, $62.5 \mathrm{kHz}$. Scan slab was set to cover the whole liver, and scanning was performed with breath hold (by expiratory stop).

\section{CT Scanner and Parameters}

In the acquisition of CT images for other comparisons, the 128-slice CT scanner SOMATOM Definition AS+ VA48A (Siemens Healthcare, Germany) was used. The imaging conditions (tube voltage, tube current) were appropriately determined by the radiologist. The optimal dose was determined depending on the patient's body size, and the image quality required for diagnosis was ensured in all patients (image noise within $\pm 10 \mathrm{HU})$.

\section{Statistical Processing}

The software used for statistical processing was EZR (64 bit, version 1.54) and Excel 2016 (Microsoft Corporation). A nonparametric test of correlation coefficients (Spearman's rank correlation coefficient) was performed to analyze the correlation coefficients between MRS-FF and DE-FF and between MRS-FF and LAI [19]. The comparison test between the samples of groups (1) and (2) was performed using unpaired t-test, and the significance test for the difference in correlation coefficient between the two groups was performed using Fisher r-to-z transformation [20].

\section{Results}

\section{Subjects}

In the subjects of this study, the mean age was $56.96 \pm 13.46$ years, the age range was $22-84$ years, the mean body mass index (BMI) was $27.04 \pm 3.51\left(\mathrm{~kg} / \mathrm{cm}^{2}\right)$, and the male:female ratio was 28:26 (Table 1). If the cutoff value of MRS-FF for intrahepatic fat quantification was set at $6.25 \%$ from a previous study, intrahepatic fat deposition was observed in 22 patients and not 32 patients [17]. 


\section{Comparison Between Fat Quantification Methods}

The mean MRS-FF of the subjects was $13.01 \% \pm 16.92 \%$, the mean DE-FF was $13.07 \% \pm 8.961 \%$, and the mean LAl was $-0.4886 \pm 12.45 \mathrm{HU}$ (Fig 3). The correlation coefficient between MRS-FF and DE-FF was $0.841(p=0.00)$, and the correlation coefficient between MRS-FF and LAI was -0.726 ( $p<0.01)$ (Fig 4). The correlation coefficients between MRS-FF and both DE-FF and LAI in previous studies were $0.892(\mathrm{p}=$ $0.00)$ and $-0.709(p<0.01)$ [12]. The difference between the correlation coefficients of Modified ${ }^{1} \mathrm{H}-\mathrm{MRS}$ and those of previous studies was determined to be significant by Fisher r-to-z transformation $(p=0.250$ [between MRS-FF and DE-FF], $p=0.849$ [between MRS-FF and LAI]), and no significant difference was found in both cases.

\section{Subjects of the Subgroup Analysis}

The subgroup analysis showed that there were 39 patients in group (1) with a BHT of $23 \mathrm{~s}$ and 15 patients in group (2) with a BHT between 14 and $22 \mathrm{~s}$. The mean age of group (1) was $55 \pm 12.18$ years, mean BMI was $27.24 \pm 3.83\left(\mathrm{~kg} / \mathrm{cm}^{2}\right)$, and the male:female ratio was 20:19. The mean age of group (2) was $62.07 \pm 15.63$ years, the mean BMI was $26.55 \pm 2.55\left(\mathrm{~kg} / \mathrm{cm}^{2}\right)$, and the male:female ratio was 8:7 (Table 2). If the cutoff value of MRS-FF for intrahepatic fat quantification was set at $6.25 \%$ from previous studies, intrahepatic fat deposition was found in 16 cases in group (1) and not 23 cases and 6 cases in group (2) and not 9 cases [17].

\section{Comparison Between Fat Quantification Methods by Subgroup Analysis}

A comparison test for MRS-FF, DE-FF, and LAI between the subgroups of groups (1) and (2) was performed $(p=0.93, p=0.86$, and $p=0.63$, respectively), and there was no significant difference in all (Fig 5). In the subgroup analysis, the correlation coefficient between MRS-FF and DE-FF in group (1) was $0.849(p=0.00)$, and that between MRS-FF and LAI was $-0.733(p=0.00)$, while, for group (2), the correlation coefficients were $0.886(p=0.00)$ and $-0.761(p=0.00)$, respectively. The difference between the correlation coefficients of groups (1) and (2) was significant by Fisher r-to-z transformation $(p=0.653$ [between MRS-FF and DE-FF], $p=0.849$ [between MRS-FF and LAI]), and no significant difference was found in both groups (Figs. 6 and 7).

\section{Discussion}

The correlation coefficients between MRS-FF, DE-FF, and LAI in the previous study and that in the Modified

${ }^{1} \mathrm{H}$-MRS were determined by Fisher $\mathrm{r}$-to-z transformation. There was no significant difference between the correlation coefficients of the two methods ( $p=0.250$ and $p=0.849$, respectively). Therefore, the reliability of MRS-FF by modified ${ }^{1} \mathrm{H}$-MRS imaging was verified. In this method, the dummy sets were set to OTR, but the recommended default by the vendor is $4 T R$. In ${ }^{1} \mathrm{H}$-MRS as head tumors, the dummy sets are the parameters used to suppress the signal of surrounded and inherent water and stabilize the signal detection of metabolite with a small SI of approximately $15 \mathrm{mM}$ [21]. Because there is little SI difference 
between various metabolites, measurement errors can easily occur if the acquired signal is unstable [22]. Conversely, water and lipid, which are the targets of this study, detected specter data with signal intensities as high as $35 \mathrm{M}$ compared to metabolites. Moreover, the scanning parameters used in this study were short TR (1149.0 ms) because breath holding should be performed during scanning. This causes the longitudinal magnetization of the water to not sufficiently recover, and the AUP of the water becomes smaller with each successive frame. Therefore, increasing the number of dummy sets may overestimate the MRS-FF because the AUP of water detected by the reference datasets becomes smaller $[23,24]$. These results suggest that there was no difference between our results and those of previous studies even if the dummy sets are set to OTR in this study.

The reason for the nonsignificant difference in the results of the subgroup analysis was the use of Sage version 7.1 (GE Healthcare) as the analysis software, which can calculate MRS-FF by extracting only the reference datasets: 2TR. The modified ${ }^{1} \mathrm{H}-\mathrm{MRS}$ used in this study consisted of the following parameters: presaturation pulse, 10TR; reference datasets, 2TR; and datasets, 8TR. The presaturation pulse of 10TR saturated the signal, and it was a parameter that is performed internally by the application so that it surrounds the setting voxel of ${ }^{1} \mathrm{H}-\mathrm{MRS}$ and could not be set to a value $<10 \mathrm{TR}$. This presaturation pulse was necessary to suppress the influx of water components from outside the setting voxel of ${ }^{1} \mathrm{H}-\mathrm{MRS}$ [21]. Therefore, to use the AUP of water and AUP of lipid for the calculation of MRS-FF, the data of the unsuppressed signal of water was an essential parameter. In the PRESS method, two ${ }^{1} \mathrm{H}-\mathrm{MRS}$ data with specter peaks of water and fat were obtained in this reference datasets: 2TR. In contrast, datasets of 8TR obtains data of suppressed water signal for metabolites, which is unnecessary for intrahepatic fat quantification. From these findings, only the reference datasets that obtain the data of the unsuppressed water signal were used to calculate the MRS-FF, while the datasets that obtain the data of the suppressed water signal were not needed. Therefore, even if breath holding was canceled during the acquisition of datasets during the imaging time, it was suggested that there was no significant difference in the results of the MRS-FF analysis.

This study has three limitations. First, we did not perform a correlation study with histopathological evaluation by liver biopsy. The reason for the first limitation was that this was a retrospective study and liver biopsy was not performed in all included patients. Moreover, the gastroenterologists at our hospital understood that MRS-FF analysis by ${ }^{1} \mathrm{H}$-MRS was a gold standard alternative to histopathological evaluation and chose to use this method because it is noninvasive for patients. Second, the study was performed only with the GE 1.5-Tesla MRI and not with other vendors or magnetic field strengths. The reason for second limitation was that the only MRI system owned by our hospital was a general-purpose GE 1.5-Tesla MR scanner. Third, only single-voxel analysis was used in this study, not multivoxel analysis. This was because the time required for multivoxel ${ }^{1} \mathrm{H}$-MRS imaging was far beyond the range of a single breath holding and thus would lead to a respiratory-induced motion artifact. Therefore, it was not addressed in this study, which aims to evaluate it with the minimum number of ${ }^{1} \mathrm{H}-\mathrm{MRS}$ specter data and minimum BHT required [16]. Our results showed that the minimum required BHT was shorter than that of the conventional method (displayed value) using ${ }^{1} \mathrm{H}$-MRS for intrahepatic fat determination by the PRESS 
method, which is generally recognized as time-consuming. Our results suggest that this method can be extended to patients who have difficulty in performing longer breath hold. The expanded scope of the ${ }^{1} \mathrm{H}-$ MRS is expected to be used for liver screening to detect fat deposition in the liver at an early stage by clinical examination in more hospitals that have MRI systems. However, training of the technologist is essential because the examination can be completed in a shorter time than the number of times displayed as the scanning time.

\section{Conclusion}

The correlation coefficients between MRS-FF and DE-FF and between MRS-FF and LAI were strongly correlated using Modified ${ }^{1} \mathrm{H}$-MRS and Sage (for ${ }^{1} \mathrm{H}$-MRS analysis application). These results were not significantly different from the correlation coefficients of previous studies, suggesting that shortening the $\mathrm{BHT}$ requirement from 14 to $22 \mathrm{~s}$ compared with $23 \mathrm{~s}$ did not affect the ${ }^{1} \mathrm{H}$-MRS analysis results.

\section{Abbreviations}

NAFLD: non-alcoholic fatty liver disease

DE: dual echo

LAl: liver attenuation index

${ }^{1} \mathrm{H}-\mathrm{MRS}$ : proton-magnetic resonance spectroscopy

MRS-FF: MRS fat fraction

PRESS: point resolved spectroscopy

ROI: region of interest

AUP: area under the peak

IN: in-phase

OP: opposed-phase

DE-FF: dual echo-fat fraction

SI: signal intensity

FS: fat saturation

HU: Hounsfield units

BHT: breath-hold time 
TR: repetition time

TE: echo time

STEAM: stimulated echo acquisition mode

SNR: signal-to-noise ratio

\section{Declarations}

\section{Acknowledgment:}

We would like to thank the staff at the Radiology Department at Tokai University Oiso Hospital and many radiologists and radiological technologists in Japan who provided us with advice.

The authors would like to thank Enago (www.enago.jp) for the English language review.

\section{Funding:}

The author(s) received no financial support for the research, authorship, and/or publication of this article.

\section{Conflicts of interest/Competing interests:}

All other authors have no conflicts of interest.

\section{Availability of data and material:}

Data available on request due to privacy/ethical restrictions.

\section{Code availability:}

'Not applicable'

\section{Ethics approval}

This retrospective study was approved by the Institutional Review Board of Tokai University Oiso Hospital (IRB number: No. 20R-399).

\section{Consent to participate}

Informed consent was waived from all patients by the IRB.

\section{Consent for publication}

Informed consent was waived from all patients by the IRB. 


\section{References}

[1] Tateishi R, et al. Clinical characteristics, treatment, and prognosis of non-B, non-C hepatocellular carcinoma: a large retrospective multicenter cohort study. Journal of Gastroenterology volume 50, pages350-360(2015)

[2] Tateishi R, et al. A nationwide survey on non-B, non-C hepatocellular carcinoma in Japan: 2011-2015 update. J Gastroenterol 2018. doi: 10.1007/s00535—018-1532—5.

[3] Tateishi R, et al. Liver Cancer Background Trends: Increasing Non-B Non-C Liver Cancer. Internal Medicine Volume 123, Issue 5 (May 2019), 1053 - 1058.

[4] Dulai PS, et al. Increased risk of mortality by fibrosis stage in nonalcoholic fatty liver disease: Systematic review and meta-analysis. Hepatology 65: 1557-1565, 2017. doi: 10.1002/hep.29085. Epub 2017 Mar 31.

[5] Kodama T, et al. Molecular profiling of nonalcoholic fatty liver disease-associated hepatocellular carcinoma using SB transposon mutagenesis. Proc Natl Acad Sci U S A 115: E10417-10426, 2018. doi: 10.1073/ pnas.1808968115. Epub 2018 Oct 16.

[6] Eguchi $\mathrm{Y}$, Hyogo H, Ono M, et al: Prevalence and associated metabolic factors of nonalcoholic fatty liver disease in the general population from 2009 to 2010 in Japan: a multicenter large retrospective study. J Gastroenterol 2012; 47: 586-595.

[7] Omagari K, Morikawa S, Nagaoka S, et al: Predictive factors for the development or regression of Fatty liver in Japanese adults. J Clin Biochem Nutr 2009; 45: 56-67.

[8] Takahashi $\mathrm{H}$, Ono M, Hyogo H, et al: Biphasic effect of alcohol intake on the development of fatty liver disease. J Gastroenterol 2015; 50: 1114-1123.

[9] Gore RM. Diffuse liver disease. In: Gore RM, Levine MS, Laufer I, eds. Textbook of Gastrointestinal Radiology. Philadelphia: Saunders,1994; 1968-2017.

[10] Yajima Y, Ohta K, Narui T, Abe R, Suzuki H, Ohtsuki M. Ultrasonographical diagnosis of fatty liver: significance of the liver-kidney contrast. Tohoku J. Exp. Med. 1983; 139: 43-50.

[11] Fusamoto $\mathrm{H}$, Suzuki K, Hayashi $\mathrm{N}$ et al. Obesity and liver disease: evaluation of fatty infiltration of the liver using ultrasonicattenuation. J. Nutr. Sci. Vitaminol. Tokyo 1991; 37 (Suppl.): S71-7

[12] Rastogi et al. Comparative accuracy of CT, dual-echo MRI and MR spectroscopy for preoperative liver fat quantification in living related liver donors. The Indian Journal of Radiology \& Imaging, 01 Jan 2016, 26(1):5-14 
[13] Mazen Noureddin, et al. Utility of Magnetic Resonance Imaging Versus Histology for Quantifying Changes in Liver Fat in Nonalcoholic Fatty Liver Disease Trials. Hepatology. 2013 December; 58(6): 1930-1940. doi:10.1002/hep.26455.

[14] Bo-kyeong Kang, et al. Feasibility of modified Dixon MRI techniques for hepatic fat quantification in hepatic disorders: validation with MRS and histology. The British Journal of Radiology, Volume 91, Issue 1089, September 2018

[15] Mary E. Rinella, et al. Dual-echo, chemical shift gradient-echo magnetic resonance imaging to quantify hepatic steatosis: Implications for living liver donation. Liver Transplantation, Vol 9, No 8 (August), 2003: pp 851-856

[16] Scott B, et al. Quantitative assessment of liver fat with magnetic resonance imaging and spectroscopy. Journal of Magnetic Resonance Imaging, Volume 34, Issue 4 p. 729-749

[17] Janakan Satkunasingham, et al. Liver fat quantification: Comparison of dual-echo and triple-echo chemical shift MRI to MR spectroscopy. European Journal of Radiology, Volume 84, Issue 8, August 2015, Pages 1452-1458.

[18] Anneloes E. Bohte, et al. The diagnostic accuracy of US, CT, MRI and ${ }^{1} \mathrm{H}-\mathrm{MRS}$ for the evaluation of hepatic steatosis compared with liver biopsy: a meta-analysis. Eur Radiol (2011) 21:87-97

[19] Bone Marrow Transplantation 2013:48,452-458

[20] Silver, N. C., \& Dunlap, W. P. (1987). Averaging correlation coefficients: Should Fisher's z transformation be used? Journal of Applied Psychology, 72(1), 146-148.

[21] Isobe T, et al. Essentials of Brain MRS: Fundamentals and Clinical Applications. Jpn. J. Med. Phys. Vol. 36 No. 2: 85-91 (2016)

[22] Adrianus J. Bakermans, et al. In vivo proton T1 relaxation times of mouse myocardial metabolites at 9.4T. Magnetic Resonance in Medicine 73:2069-2074 (2015)

[23] Gavin Hamilton, PhD, et al. Effect of PRESS and STEAM Sequences on Magnetic Resonance Spectroscopic Liver Fat Quantification. JOURNAL OF MAGNETIC RESONANCE IMAGING 30:145-152 (2009)

[24] Carsten Thomsen, et al. Quantification of liver fat using magnetic resonance spectroscopy. Magnetic Resonance Imaging. Volume 12, Issue 3, 1994, Pages 487-495

[25] Gavin Hamilton PhD, et al. Effect of PRESS and STEAM sequences on magnetic resonance spectroscopic liver fat quantification. Journal of Magnetic Resonance Imaging, Volume 30, Issue $1 \mathrm{p}$. 145-152 


\section{Tables}

Table 1. Subject features

\begin{tabular}{|c|c|c|}
\hline \multicolumn{3}{|l|}{ Age } \\
\hline & Average (years) & $56.96 \pm 13.46$ \\
\hline & Range (years) & $22-84$ \\
\hline Sex & & $(n=54)$ \\
\hline & Male & 28 \\
\hline & Female & 26 \\
\hline \multicolumn{3}{|l|}{ BMI } \\
\hline & Average $\left(\mathrm{kg} / \mathrm{cm}^{2}\right)$ & $27.04 \pm 3.51$ \\
\hline & Range $\left(\mathrm{kg} / \mathrm{cm}^{2}\right)$ & $21.64-35.43$ \\
\hline \multicolumn{3}{|l|}{ MRS-FF } \\
\hline & Average (\%) & $13.01 \pm 16.92$ \\
\hline & Range (\%) & $0.3249-62.87$ \\
\hline \multicolumn{3}{|l|}{ DE-FF } \\
\hline & Average (\%) & $13.07 \pm 8.961$ \\
\hline & Range (\%) & $0.4793-37.66$ \\
\hline \multicolumn{3}{|l|}{ LAI } \\
\hline & Average (HU) & $-0.4886 \pm 12.45$ \\
\hline & Range (HU) & $-40.4-22.5$ \\
\hline
\end{tabular}

Table 2. Features of subject in subgroup analysis 

(1) $\mathrm{BHT}=23 \mathrm{~s}$
(2) $14 \leqq \mathrm{BHT}<23 \mathrm{~s}$

\begin{tabular}{|c|c|c|c|}
\hline \multicolumn{4}{|l|}{ Age } \\
\hline & Average (years) & $55 \pm 12.18$ & $62.07 \pm 15.63$ \\
\hline & Range (years) & $22-69$ & $22-84$ \\
\hline Sex & & $(n=39)$ & $(n=15)$ \\
\hline & Male & 20 & 8 \\
\hline & Female & 19 & 7 \\
\hline \multicolumn{4}{|l|}{ BMI } \\
\hline & Average $\left(\mathrm{kg} / \mathrm{cm}^{2}\right)$ & $27.24 \pm 3.83$ & $26.55 \pm 2.55$ \\
\hline & Range $\left(\mathrm{kg} / \mathrm{cm}^{2}\right)$ & $21.64-35.43$ & $22.70-31.22$ \\
\hline \multicolumn{4}{|l|}{ MRS-FF } \\
\hline & Average (\%) & $12.21 \pm 16.22$ & $12.63 \pm 15.51$ \\
\hline & Range (\%) & $0.3717-62.87$ & $0.3249-38.19$ \\
\hline \multicolumn{4}{|l|}{ DE-FF } \\
\hline & Average (\%) & $12.77 \pm 9.027$ & $13.07 \pm 6.726$ \\
\hline & Range (\%) & $0.4793-37.66$ & $4.975-25.04$ \\
\hline \multicolumn{4}{|l|}{ LAl } \\
\hline & Average (HU) & $-1.058 \pm 13.38$ & $0.7173 \pm 11.43$ \\
\hline & Range (HU) & $-40.4-22.5$ & $-26.3-13.60$ \\
\hline
\end{tabular}

Figures 


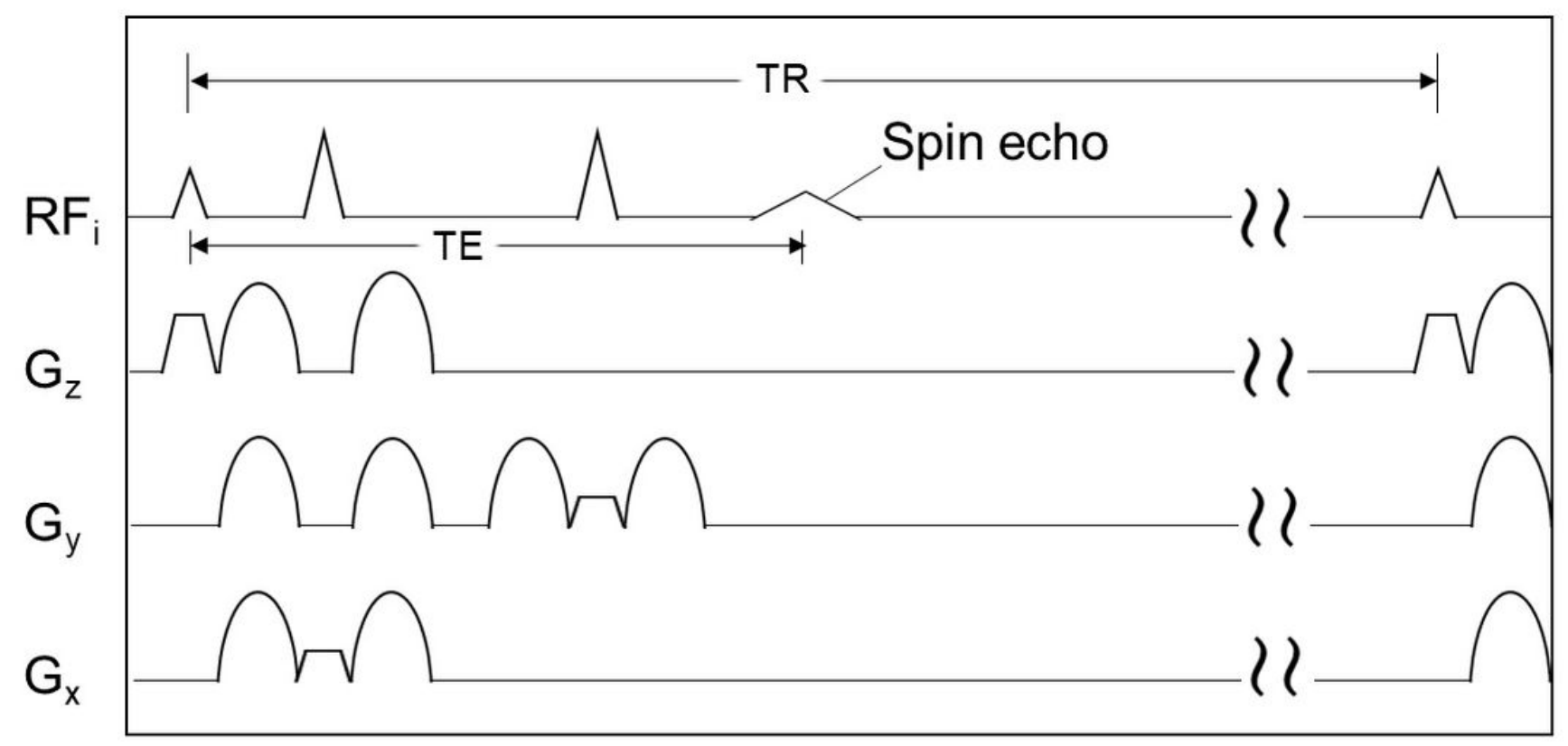

Time[msec]

Figure 1

Pulse sequence diagram of $1 \mathrm{H}-\mathrm{MRS}$ using PRESS technique. Figure 1 is a pulse sequence diagram of 1TR of $1 \mathrm{H}-\mathrm{MRS}$ imaging using the PRESS method. After one excitation pulse and two refocusing pulses are applied, a spin echo is generated, and MRS specter data are acquired.

Pre-saturation dummy sets data sets

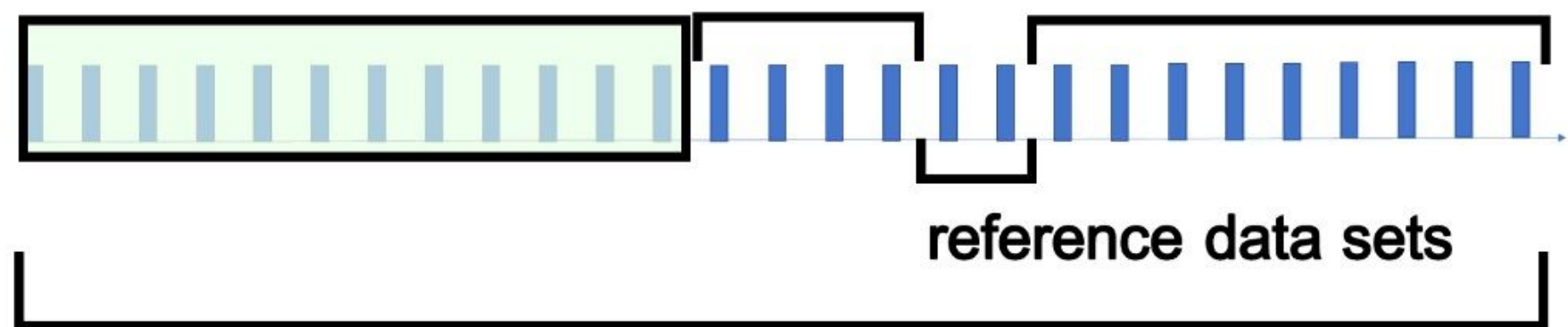

Total scan time

Figure 2

I $: 1$ Frame time $=$ TR $[\mathrm{msec}]$ 
Overview of total scan time with the PRESS technique by combining any frames (presaturation, dummy sets, reference datasets, and datasets). Figure 2 shows the concept for calculating the total scan time of $1 \mathrm{H}-M R S$ using the PRESS method. One TR is one frame in the PRESS method, which is a single-shot method, and one specter data are acquired. Presaturation, dummy sets, reference datasets, and datasets are scanned in this order. The sum of these is the total scanning time.

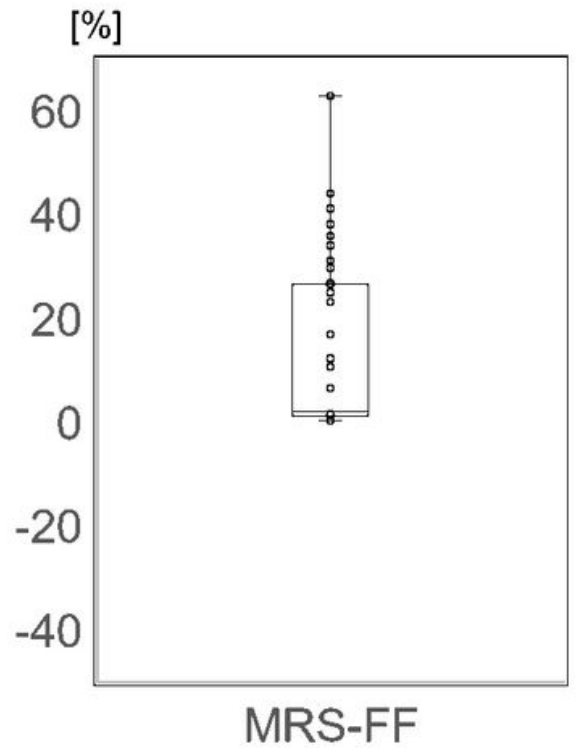

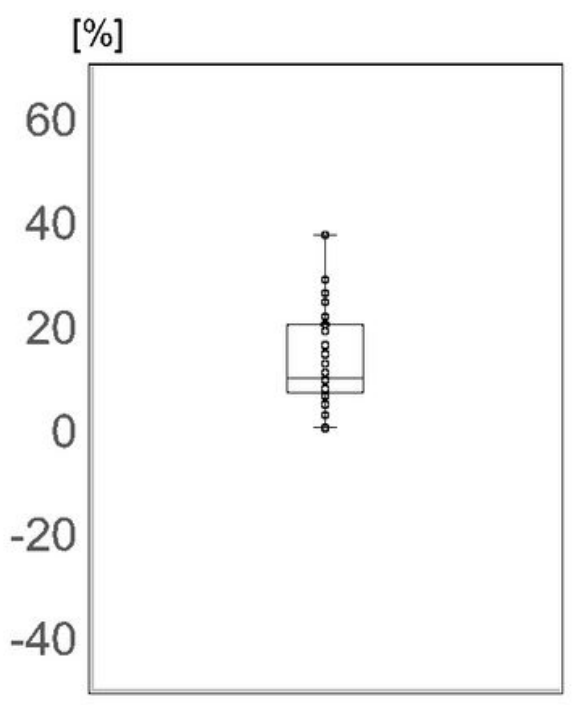

DE-FF

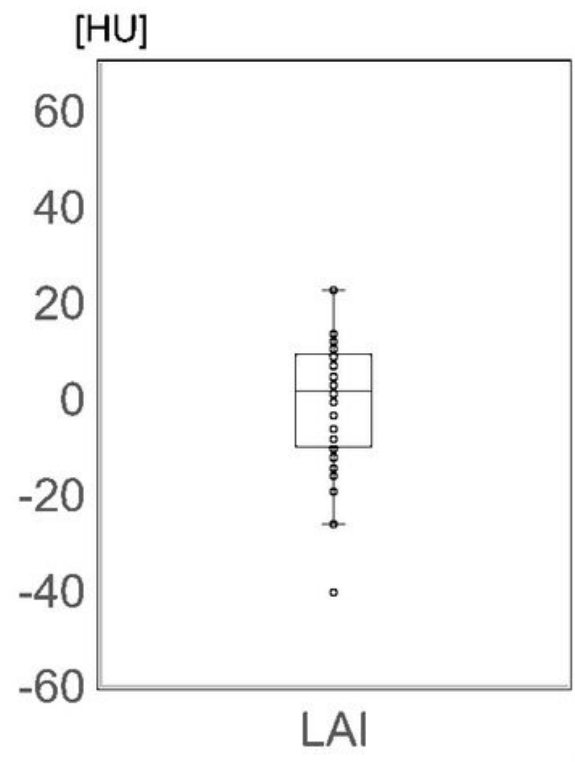

\section{Figure 3}

Distribution of each measurement results (MRS-FF, DE-FF, and LAI). Figure 3 shows the distribution of the measured MRS-FF, DE-FF, and LAI results for all subjects in the graph. The graph shows the maximum, minimum, and median values in a box plot.
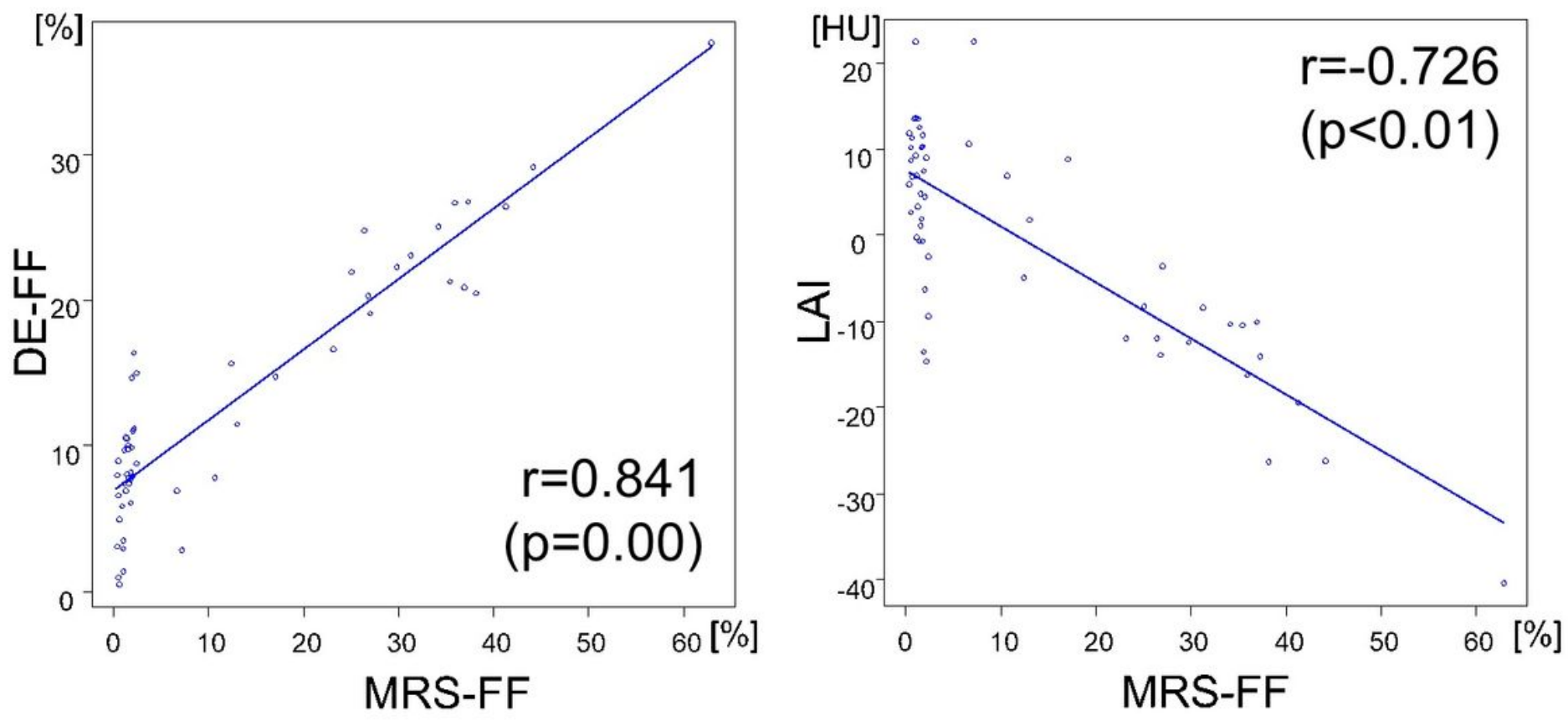

Figure 4 
Correlation between MRS-FF and DE-FF (left) and MRS-FF and LAI (right). Figure 4 shows the horizontal axis of MRS-FF [\%] and vertical axis of DE-FF [\%] (left) and LAI [HU] (right), showing the strong correlation between them. MRS-FF and DE-FF had a positive correlation with a correlation coefficient of $0.841(p=$ $0.00)$. MRS-FF and LAl had a negative correlation with a correlation coefficient of $-0.726(p<0.01)$.

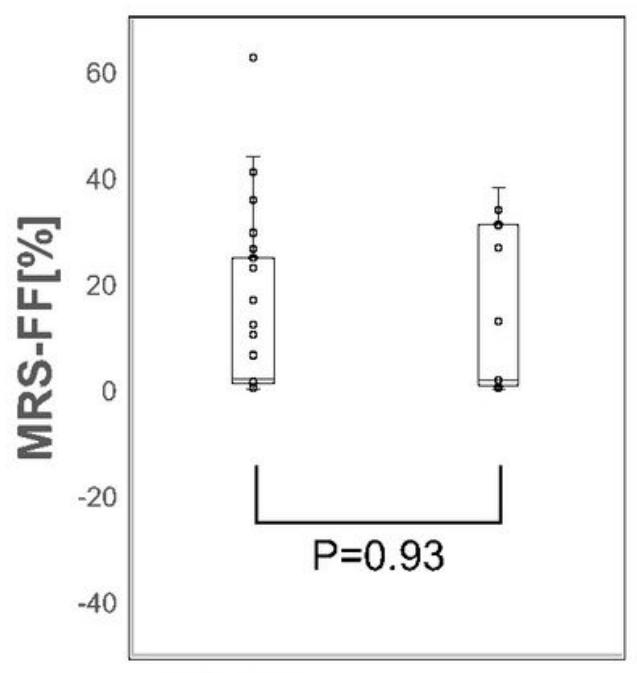

(1) $\mathrm{BHT}=23 \mathrm{sec}$

(2) $14 \leqq$ BHT $<23$ sec

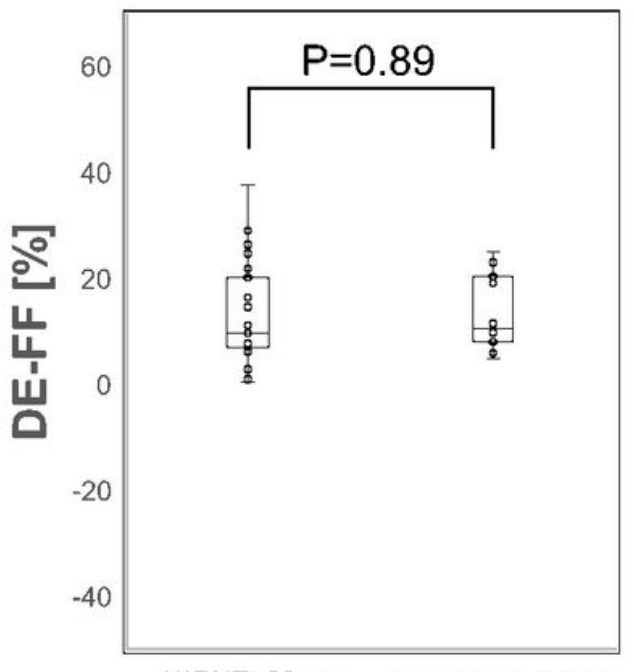

(1) $\mathrm{BHT}=23 \mathrm{sec}$

(2) $14 \leqq$ BHT $<23$ sec

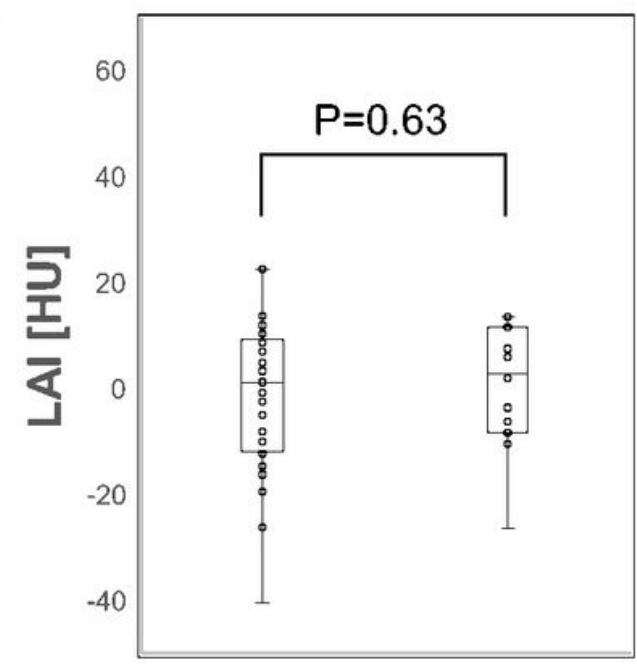

(1) $\mathrm{BHT}=23 \mathrm{sec} \quad$ (2) $14 \leqq \mathrm{BHT}<23 \mathrm{sec}$

Figure 5

Comparison of distribution in each measurement results (MRS-FF, DE-FF, and LAI) in the subgroup analysis (comparison by "BHT $=23 \mathrm{~s}$ " or " $14 \leqq \mathrm{BHT}<23$ "). Figure 5 shows the results of comparing the distribution of MRS-FF, DE-FF, and LAI by subgroup analysis. The horizontal axis of all graphs shows the category of the subgroup, with group (1) BHT = $23 \mathrm{~s}$ on the left and group (2) BHT = 14-22 s on the right. The left is MRS-FF [\%] on the vertical axis and shows that there was no significant difference in the distribution between subgroups ( $p=0.93)$. The center is DE-FF [\%] on the vertical axis, and there was no significant difference in the distribution between subgroups $(p=0.89)$. The right is LAI [HU] on the vertical axis, and there was no significant difference in the distribution between subgroups $(p=0.63)$. 


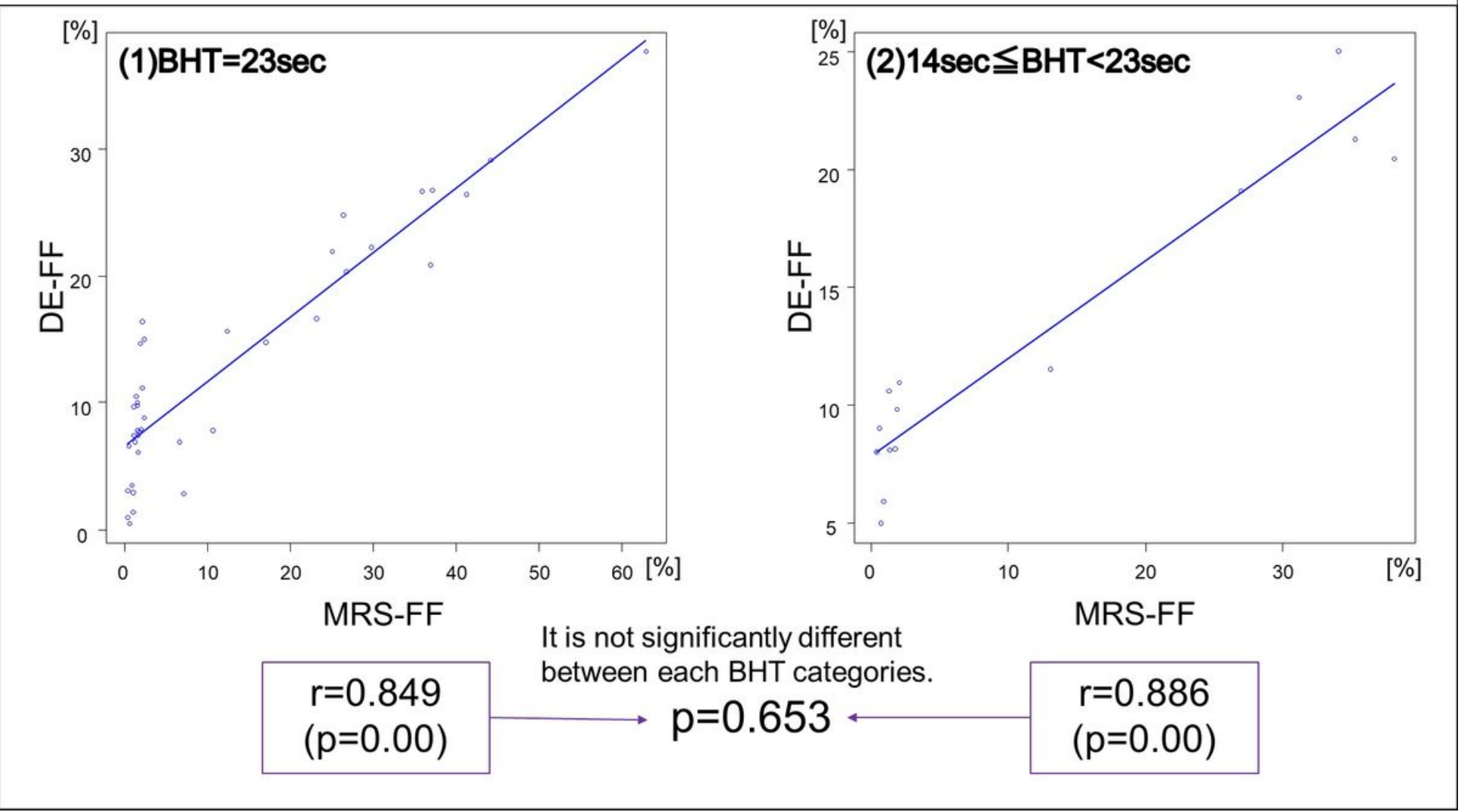

Figure 6

Correlation between MRS-FF and DE-FF from group (2) is not significantly different from group (1). Figure 6 shows the horizontal axis of MRS-FF [\%] and vertical axis of DE-FF [\%], showing the strong correlation between them. The left shows the result of subgroup (1) BHT = $23 \mathrm{~s}$, and the correlation coefficient was $0.849(p=0.00)$. The right shows the results of subgroup (2) BHT $=14-22 \mathrm{~s}$, and the correlation coefficient was $0.886(p=0.00)$. The results of Fisher r-to-z transformation on both correlation coefficients showed that there was no significant difference between the subgroups $(p=0.653)$. 


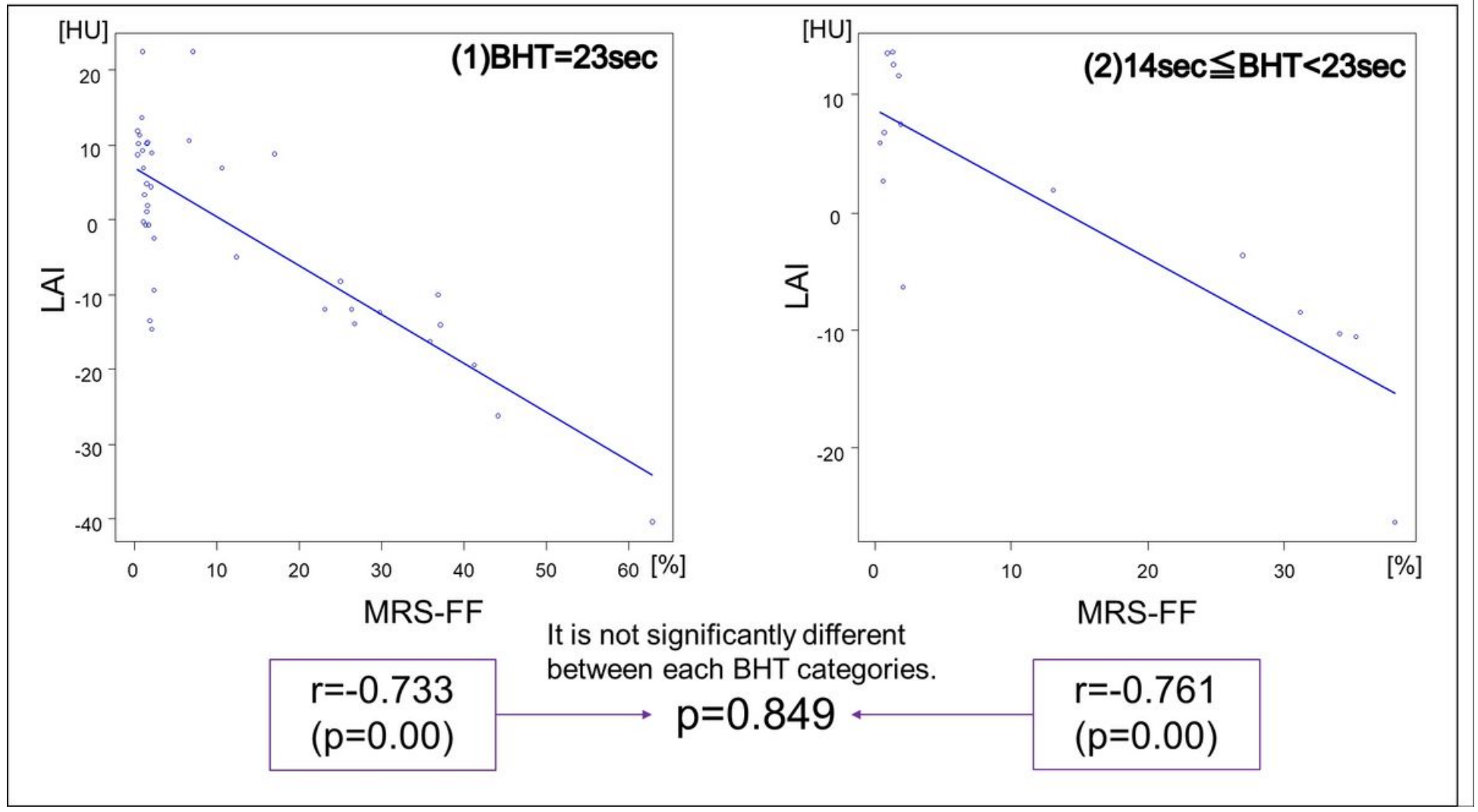

\section{Figure 7}

Correlation between MRS-FF and LAl from group (2) is not significantly different from group (1). Figure 7 shows the horizontal axis of MRS-FF [\%] and vertical axis of LAI [HU], showing the strong correlation between them. The left shows the result of subgroup (1) BHT $=23 \mathrm{~s}$, and the correlation coefficient was $-0.733(p=0.00)$. The right shows the results of subgroup (2) BHT $=14-22 \mathrm{~s}$, and the correlation coefficient was $-0.761(p=0.00)$. The results of Fisher $r-$ to-z transformation on both correlation coefficients showed that there was no significant difference between the subgroups, $p=0.849$. 